\title{
The Educational Impact of the Specialty Care Access Network-Extension of Community Healthcare Outcomes Program
}

\author{
Reena J. Salgia, $M D_{,}^{1, *}$ Patricia B. Mullan, $P h D_{1}{ }^{2}$ \\ Heather McCurdy, BSN, Anne Sales, PhD, ${ }^{3,4}$ \\ Richard H. Moseley, MD, ${ }^{1,3}$ and Grace L. Su, MD ${ }^{1,3}$ \\ Departments of ${ }^{1}$ Internal Medicine and ${ }^{2}$ Medical Education, \\ University of Michigan Medical School, Ann Arbor, Michigan. \\ ${ }^{3}$ VA Ann Arbor Healthcare System, Ann Arbor, Michigan. \\ ${ }^{4}$ VA Center for Clinical Management Research, Ann Arbor, \\ Michigan. \\ *Present address: Henry Ford Hospital, Detroit, Michigan.
}

\section{Abstract}

Background: With the aging hepatitis C cohort and increasing prevalence of fatty liver disease, the burden on primary care providers (PCPS) to care for patients with liver disease is growing. In response, the Veterans Administration implemented initiatives for primary care-specialty referral to increase PCP competency in complex disease management. The Specialty Care Access Network-Extension of Community Healthcare Outcomes (SCAN-ECHO) program initiative was designed to transfer subspecialty knowledge to PCPs through case-based distance learning combined with real-time consultation. There is limited information regarding the initiative's ability to engage PCPs to learn and influence their practice. Materials and Methods: We surveyed PCPs to determine the factors that led to their participation in this program and the educational impact of participation. Results: Of 51 potential participants, 24 responded to an anonymous survey. More than $75 \%$ of respondents participated more than one time in a SCAN-ECHO clinic. Providers were motivated to participate by a desire to learn more about liver disease, to apply the knowledge gained to future patients, and to save their patients time traveling to another center for specialty consultation. Seventy-one percent responded that the didactic component and case-based discussion were equally important. It is important that participation changed clinical practice: $75 \%$ of providers indicated they had personally discussed the information they learned from the case presentations with their colleague(s), and 42\% indicated they helped a colleague care for their patient with the knowledge learned during discussions of other participants' cases. Conclusions: This study shows that the SCAN-ECHO videoconferencing program between PCPs and specialists can educate providers in the delivery of specialty care from a distance and potentially improve healthcare delivery.

Key words: telemedicine, telehealth, telecommunications, distance learning

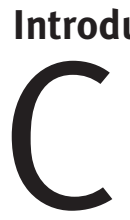

Introduction patients and providers in rural and underserved areas, access to specialty care consultation may be limited. Primary care providers (PCPs) are often faced with having to care for patients with complex chronic liver disease on their own. This is due to large geographic distances to tertiary-care centers and the absence of liver specialists in rural and underserved areas. This problem has been recognized within the Veterans Health Administration (VHA) and is being addressed by a wide range of innovative initiatives to transform the present model of care. One such program, Specialty Care Access Network (SCAN)-Extension of Healthcare Outcomes (ECHO), was implemented in May 2011. Based on the Project ECHO model developed at the University of New Mexico, ${ }^{3,4}$ the VHA's SCAN-ECHO program sought to use video-teleconferencing between primary providers and specialists to provide case-based distance learning via continuing medical education (CME) programs to PCPs in rural and underserved area. Compared with the traditional problem-based learning CME programs, the SCANECHO program provides real-time specialty consultation at the time of the session.

As a pilot demonstration site for SCAN-ECHO, the Veterans Administration (VA) Ann Arbor Healthcare System (VAAAHS) implemented the first SCAN-ECHO liver clinic/conferences, Ann Arbor SCAN-ECHO Liver. PCPs submitted their questions to the SCAN-ECHO Liver "clinic" in the same electronic method as they would request a traditional face-to-face consultation, by use of the VHA computerized patient records system. Cases are then scheduled into the conference, presented by the PCPs, and discussed by video-teleconference twice per month. In addition, a prerecorded didactic session on liver-specific topics tailored for PCPs is also presented.

Case-based learning is a powerful teaching tool in medical school education, but less is known about it in the context of postgraduate CME programs. ${ }^{5,6}$ Distance learning has also been shown to be an effective method of learning in many educational systems. ${ }^{7}$ What is not known is how the combination of real-time consultation and distance learning is perceived by PCPs. In this study, we aimed to determine the educational impact of this novel program on PCPs and evaluate factors that may impact provider participation. 


\section{EDUCATIONAL IMPACT OF SCAN-ECHO TELEMEDICINE PROGRAM}

\section{Materials and Methods}

During the period of June 2011 (initiation of the program) to September 2012, 55 participating PCPs presented 203 cases (representing 183 unique patients). The average participation was 2.5 SCAN-ECHO clinics per PCP (range, 1-17). Of the 55 PCPs, only 51 were still employed within the VA Healthcare System and available at the time of survey. These PCPs were from 14 sites that were located within four different VA Healthcare Systems and their affiliate community-based outpatient clinics. All of the consults originated from the liver clinic consult requests where patients would have otherwise been asked to travel to VAAAHS to seek specialist consultation. PCPs did not directly receive monetary reward for participating in the SCAN-ECHO clinic; however, they did have the opportunity to receive CME credit.

\section{STUDY DESIGN AND PARTICIPANTS}

This study involved PCPs through the VHA in Veterans Integrated Service Network 11, which covers Michigan, Indiana, northwestern Ohio, and east-central Illinois. The VA Ann Arbor Medical Center is a tertiary-care center with specialists in liver disease participating in the SCAN-ECHO program. PCPs including general internists, nurse practitioners, and physician assistants are located at VA Ann Arbor and community-based outpatient clinics within this Veterans Integrated Service Network. General internists are adult internal medicine physicians independently licensed to practice medicine. Nurse practitioners and physician assistants are advanced practitioners working in conjunction with physicians. They have the ability to focus their practice within a specialty. Through their VA license, nurse practitioners and physician assistants see patients independently, however, under supervision of a physician.

Providers who presented a patient for case discussion at a SCANECHO videoconferencing session were eligible to participate. In order to collect the most representative data regarding participation, surveys were intentionally not sent to providers who did not present cases. Electronic surveys were distributed to providers and collected through Qualtrics ${ }^{\circledR}$ software (www.qualtrics.com). All responses were anonymous. The survey instrument contained 21 choice questions and 3 free-text questions. Responders were asked to select from multiple answer choices for each question and had the ability to select multiple applicable answers. Questions related to motivating factors, barriers to participation, time spent participating, and application of learned content had descriptive options to choose from in order to best understand the issues affecting participation. The survey was tested among a peer group prior to distribution. Approval was received by the VAAAHS Institutional Review Board to conduct this study.

\section{MEASUREMENTS AND ANALYSIS}

Data were collected and grouped into themes to describe the factors influencing participation in this program, educational impact, value and feasibility, and the likelihood of changing future practice in caring for patients with similar types of illness. Descriptive statistics were used to describe the demographic characteristics of the study participants.

\section{Results \\ PARTICIPANT DEMOGRAPHICS}

Fifty-one PCPs received the electronic survey, and 24 (47\%) responded. The completion rate of the survey was very high at $96 \%$ for all choice questions. Among the participants, providers included 13 (54\%) physicians, 4 (17\%) physician assistants, and 7 (29\%) nurse practitioners. Physicians were certified in either general internal medicine or family practice. The physician assistants and nurse practitioners were focused in primary care clinic settings. Seventeen participants $(71 \%)$ responded that they see $>40$ patients per week in clinic. Of note is that the proportion of these patients with liver disease (acute or chronic) was $>5 \%$ for 16 (67\%) PCPs, and for 5 $(21 \%)$ providers, liver disease affected $>15 \%$ of their patients.

\section{FACTORS INFLUENCING PARTICIPATION}

One of the main objectives of this study was to assess the factors that both motivate and limit participation in SCAN-ECHO, as well as the feasibility of distance learning and specialty consultation through videoconferencing. We specifically asked participants in the SCAN-ECHO program about factors that motivated them to participate and barriers to their participation (Figs. 1 and 2). Participants were asked how often they had participated in a SCAN-ECHO videoconference. Of the 24 respondents, 6 (25\%) participated once, 7 (29\%) participated two or three times, and 11 (46\%) participated more than three times. Participants who engaged in more than one videoconference session either presented another unique patient case(s) or requested follow-up discussion of a previously presented patient. Of the 24 respondents, 11 (46\%) presented 1-2 unique patient cases, 8 (33\%) presented 3-5 cases, 3 (13\%) presented 6-10 cases, and 2 PCPs $(8 \%)$ presented $>10$ patient cases at these videoconferencing sessions.

The majority of participants $(n=18,75 \%)$ indicated that they were motivated to participate to learn more about liver disease, 18 (75\%) were able to apply the knowledge they gained to future patients, 18 (75\%) reported it saved their patient time traveling to another VA Medical Center for specialty consultation, 16 (67\%) participated because they believe that videoconferencing is important for the future care of patients, and 18 (75\%) desired to increase collaboration with specialists. About half of the responders $(n=11,46 \%)$ reported that saving their patient money and decreasing overall healthcare costs, preventing professional isolation ( $n=10,42 \%)$, and obtaining CME credit $(n=13,54 \%)$ were also important. Few responders $(n=7,29 \%)$ indicated that they have protected time to participate in this program. When asked the time of day that was used to participate in the videoconference, eight (33\%) responders indicated that they participated during their lunch break, eight (33\%) indicated time between patients on a clinic day, five (21\%) said they used the beginning or end of a workday, and five (21\%) used administrative time. The best 


\section{SALGIA ET AL.}

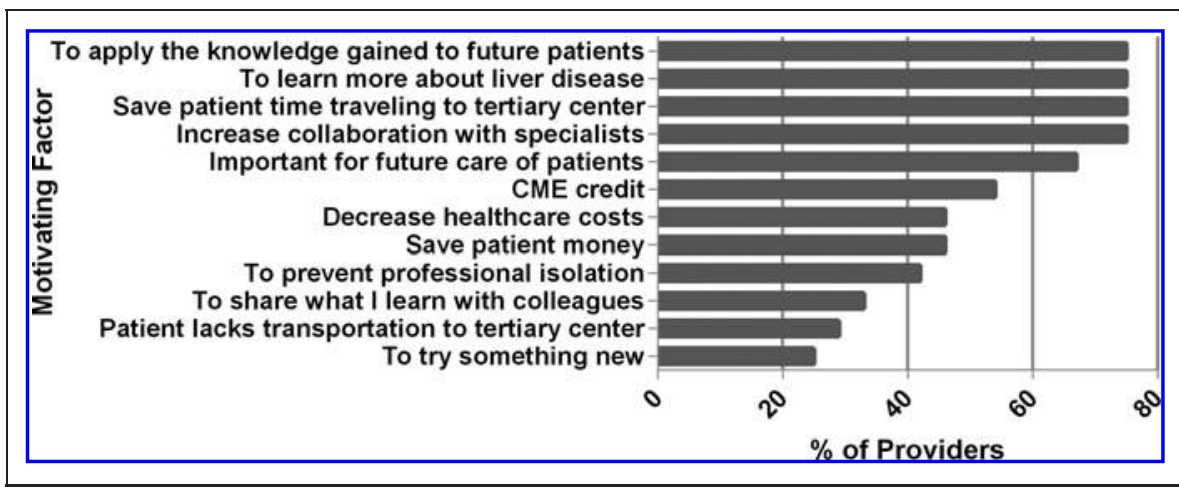

Fig. 1. Factors that motivated providers to participate $(n=24)$. CME, continuing medical education.

time of the day for these activities was suggested to be during scheduled administrative time or time free of direct patient care duties.

A majority of providers $(n=17,77 \%)$ indicated that the main barrier to participation in SCAN-ECHO was not having enough time in their schedule. Less important was receiving workload credit $(n=4$, $18 \%)$ or financial incentive $(n=4,18 \%)$ or the ability to readily access and use the technology for videoconferencing $(n=2,9 \%)$. Several providers $(n=13,54 \%)$ described incentives that would motivate them to participate more often. The most common theme was the desire for protected time to participate in the SCAN-ECHO program. Providers noted that finding time in their day to participate was very challenging, although no clear difference was noted between types of medical providers. Because of the overall limited number of participants, we are unable to determine if there are significant differences between the groups of providers in terms of motivating factors or barriers to participation.

\section{EDUCATIONAL IMPACT AND VALUE}

Participants were asked to indicate the relative importance of the case-based patient discussion and the didactic lesson, to each individual. Seventeen (71\%) providers reported equal importance of both the case-based and the didactic sessions. The remainder responded that the case-based discussion was more important, and no individual felt that the didactic session was of greater value. Currently, the didactic sessions encompass a large number of topics in liver disease. We were interested in knowing which topics the PCPs would like to learn about most during the didactic sessions. Providers ranked each of the 20 topics as "high priority" or "low priority." The five highest ranked topics were as follows: nonalcoholic fatty liver disease (or nonalcoholic steatohepatitis), general management of patients with cirrhosis, alcoholic liver disease, hepatitis C management, and hepatocellular carcinoma. If the didactic lesson was excluded, 22 (92\%) providers indicated that they were still "likely" or "very likely" to participate in SCAN-ECHO.

Despite the time constraints of the PCPs, 19 $(82 \%)$ participants indicated that they are either "likely" or "very likely" to review a didactic lecture again if it is made readily available on a Web site. Currently the lectures are all available on the VA server for the providers to access. However, we wanted to determine if there are other formats that may be desired for learning didactic information. Few providers indicated preferences other than the current online format; these included written handouts, in-person live lecture, an annual practicum in-person at the tertiary-care medical center, and a Web site with algorithms of approach to management and treatment as well as patient education materials.

\section{CHANGE OF PRACTICE}

Innovative health delivery and educational initiatives have the potential to change the practice of care providers. In the short time since SCAN-ECHO was implemented through VAAAHS, 20 (83\%) responders indicated that they had already seen other patients in the clinic with liver-related medical problems similar to the case they had personally presented at a videoconference. These same providers described how they were able to apply what they had learned to future cases (Fig. 3). The majority of them ( $n=18,90 \%)$ indicated that they were able to develop a more appropriate diagnostic approach, treatment plan ( $n=16,80 \%)$, and follow-up for the additional patients' care $(n=16,80 \%)$. Providers were asked why they might still choose to refer a patient for in-person consultation if SCAN-ECHO videoconferencing was readily available. Complexity of the disease process was indicated by 17 (71\%) responders, and patient satisfaction in seeing a specialist was indicated by 11 (46\%) responders. Less common choices included four (17\%) providers who were concerned about the perceived medical-legal consequences if the patient was not referred to a specialist and one (4\%) who indicated the need for a procedure not performed at his or her center.

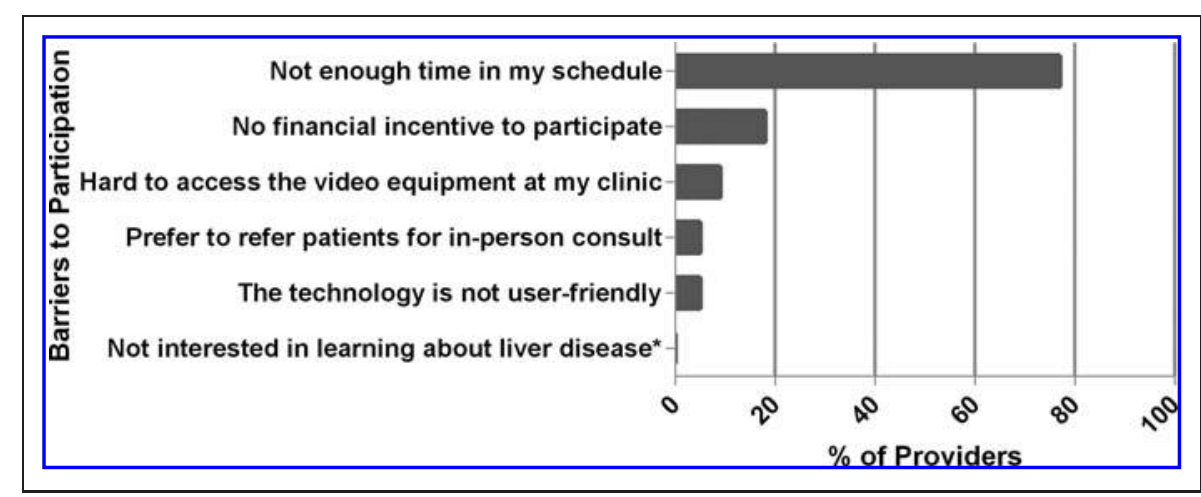

Fig. 2. Barriers to provider participation $(n=24)$. ${ }^{*}$ Indicates that $0 \%$ of providers chose this answer. 


\section{EDUCATIONAL IMPACT OF SCAN-ECHO TELEMEDICINE PROGRAM}

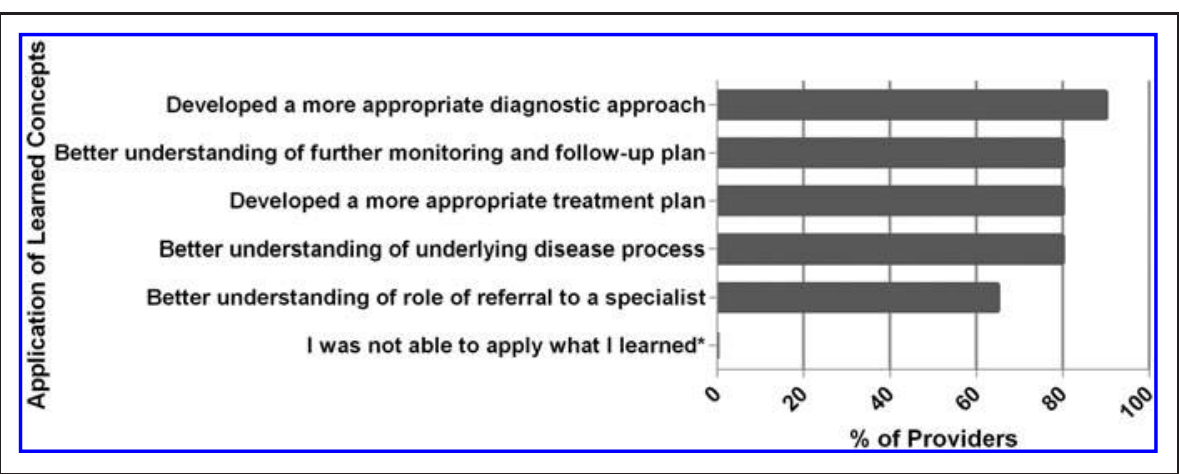

Fig. 3. The practical impact of concepts learned through the Specialty Care Access Network-Extension of Community Healthcare Outcomes Program session with additional patient encounters $(n=23)$. ${ }^{*}$ Indicates that $0 \%$ of providers chose this answer.

An unexpected finding in our results was the sharing of knowledge among providers. It is notable that 18 (75\%) providers indicated that they have personally discussed the information they learned from the didactic lesson with their colleague(s). Ten (43\%) providers indicated they helped a colleague care for his or her patient with the knowledge they learned during from the didactics. One of the providers gave a short teaching presentation to his or her colleague. Only four (17\%) providers indicated they had not yet shared this knowledge with a colleague.

Similarly, 18 (75\%) providers indicated they had personally discussed the information they learned from the case presentation(s) with their colleague(s). Ten (43\%) indicated they helped a colleague care for his or her patient with the knowledge they learned during from the case(s). Only two (8\%) providers had not yet shared this knowledge with a colleague.

All of the providers were asked how likely it is that their participation in the SCAN-ECHO session(s) has improved their ability to take care of patients with liver disease. It is notable that all 24 (100\%) responders indicated that it "likely" or "very likely" improved their ability to care for these patients. Additionally, 21 (91\%) of the providers are "likely" or "very likely" to participate in a SCAN-ECHO session again in the future.

\section{Discussion}

Based on responses from a subset of participants, our study demonstrates that distance learning through videoconferencing of patient cases and didactic sessions is feasible and desirable to a proportion of PCPs and can lead to increased provider-to-provider discussion of case management. The majority of the providers who participated in our SCAN-ECHO survey participated in the program more than once. They were motivated to participate in the videoconferencing sessions for several reasons. It is interesting that providers desired to learn more about liver disease. This is most likely because they are recognizing the increasing volume of patients with chronic liver disease. The main barrier to participation was lack of time during their workday, and many indicated that having a protected block of time would motivate them to participate more often.
Fortunately, providers indicated that they were able to utilize the knowledge they learned through the SCAN-ECHO discussions to change practice. They were able to develop more appropriate evaluation and management plans for future patients with similar disease processes and also to share the knowledge gained to assist their colleagues with management of their patient(s).

Prior studies looking at the educational impact of similar telemedicine-based initiatives have been quite limited, particularly in subspecialty internal medicine. Studies have shown that participation in telemedicine learning through a case-based format helps to foster professional development, decreases PCP iso-

lation, and increases provider satisfaction and also to build clinical knowledge and skills beyond those that are possible with traditional lectures. ${ }^{8-10}$ Similarly, several responders to our study noted that they are motivated to participate to decrease professional isolation. A randomized trial performed in Europe has shown that when compared with standard consultation, teleconsultation increased the likelihood of offering follow-up appointments, increased patient satisfaction, and reduced testing. ${ }^{11}$ Project ECHO reported survey data on provider satisfaction for 29 providers who participated in their program. They showed high rates of enhanced knowledge and competence on hepatitis C management and treatment. ${ }^{4}$ However, they did not report on motivating factors and barriers to provider participation, topics of educational interest aside from hepatitis $\mathrm{C}$, or change of practice. In 2010, they reported on effective treatment of hepatitis $\mathrm{C}$ at Project ECHO sites. $^{12}$ Although they were able to show improved treatment results for the patient, there are limited data on the educational impact, value, and feasibility of the program for the PCP.

A systematic review was recently published regarding the effectiveness of videoconsultation in clinical oncology. There were 19 studies included in the review that met study criteria. They reported on outcomes including patient satisfaction, cost of videoconsultation, patient preferences, provider satisfaction, and provider convenience. Overall, the providers showed both satisfaction for videoconsultation and reported that it was feasible. However, the studies did not investigate the educational impact or value of videoconsultation for the providers. ${ }^{13}$

It is not surprising that PCPs indicated they struggle to find time to participate in SCAN-ECHO sessions. High clinical volumes and provider shortage in many rural and medically underserved areas cause increased challenges for participation. Insufficient time and lack of reimbursement for telemedicine and videoconsultation have long been an impediment to more widespread adoption of such initiatives. ${ }^{14}$ However, our data suggest that most providers would desire to participate again if they had allocated time, as they do for standard clinic visits.

This study is limited by the common limitations of survey-design studies, including the potential for recall bias depending on the time 


\section{SALGIA ET AL.}

between provider participation in SCAN-ECHO and survey completion. Additionally, the majority of the questions in our study were choice questions, and this limits the contextual interpretation of the answers. To overcome this, providers were given the option to provide free-text responses. Survey studies are limited by the response rates. Our response rate was $47 \%$. It is likely that additional responses could have been obtained if providers had been sent directed reminders to complete the electronic survey. However, because of our anonymous approach to data collection, this was not possible. Regardless, this raises the possibility of nonresponse bias. We are unable to compare the impact of this program on the survey responders versus nonresponders. Additionally, there is potential for social desirability bias, as providers who like the SCAN-ECHO program are more likely to respond favorably in order to see it continue. Despite these limitations, this is one of the first videoconferencing distance learning programs to report on the educational impact, feasibility, and opinions of PCPs.

In conclusion, our study is unique in its focus on the educational impact of a distance learning case-based and didactic program for clinical care. Important findings of our study on the VHA SCANECHO program in liver disease include a better understanding of factors that motivate and limit participation in the program, the value of the case-based versus didactic sessions, and the unexpected finding that providers have shared the knowledge they gained with their colleagues. Sustainability of this and similar programs requires that future efforts are focused on providing resources and protected time toward the providers committed to distance learning.

\section{Disclosure Statement}

No competing financial interests exist.

\section{REFERENCES}

1. Shepard CW, Finelli L, Alter MJ. Global epidemiology of hepatitis C virus infection. Lancet Infect Dis 2005;5:558-567.

2. Kim WR, Brown RS Jr, Terrault NA, El-Serag H. Burden of liver disease in the United States: Summary of a workshop. Hepatology 2002;36:227-242.

3. Arora S, Thornton K, Jenkusky SM, Parish B, Scaletti JV. Project ECHO: Linking university specialists with rural and prison-based clinicians to improve care for people with chronic hepatitis C in New Mexico. Public Health Rep 2007;122(Suppl 2):74-77.
4. Arora S, Geppert CM, Kalishman S, Dion D, Pullara F, Bjeletich B, et al. Academic health center management of chronic diseases through knowledge networks: Project ECHO. Acad Med 2007;82:154-160.

5. McLean SF, Horn K, Tyroch AH. Case based review questions, review sessions, and call schedule type enhance knowledge gains in a surgical clerkship. $\underline{J}$ Surg Educ 2013;70:68-75.

6. Feist M, Ciccarelli M, McFerron BA, Molleston JP. Methods and effects of a casebased pediatric gastroenterology online curriculum. J Pediatr Gastroenterol Nutr 2013;56:161-155.

7. Allen $M$, Mabry E, Mattrey $M$, Bourhis J, Titsworth $S$, Burrell N. Evaluating the effectiveness of distance learning: A comparison using meta-analysis. $J$ Communication 2004;54:402-420.

8. Morrow CB, Epling JW, Teran S, Sutphen SM, Novick LF. Future applications of case-based teaching in population-based prevention. Am J Prev Med 2003; 24(4 Suppl):166-169.

9. Davis RS, Bukstein DA, Luskin AT, Kailin JA, Goodenow G. Changing physician prescribing patterns through problem-based learning: An interactive, teleconference case-based education program and review of problem-based learning. Ann Allergy Asthma Immunol 2004;93:237-242.

10. Norris $T E$, Hart GL, Larson EH, Tarczy-Hornoch P, Masuda DL, Fuller SS, et al. Low-bandwidth, low-cost telemedicine consultations in rural family practice. J Am Board Family Pract 2002;15:123-127.

11. Wallace $P$, Haines $A$, Harrison $R$, Barber J, Thompson $S$, Jacklin $P$, et al. Joint teleconsultations (virtual outreach) versus standard outpatient appointments for patients referred by their general practitioner for a specialist opinion: A randomised trial. Lancet 2002;359:1961-1968.

12. Arora S, Kalishman S, Thornton K, Dion D, Murata G, Deming P, et al. Expanding access to hepatitis $C$ virus treatment-Extension for Community Healthcare Outcomes (ECHO) project: Disruptive innovation in specialty care. Hepatology 2010;52:1124-1133.

13. Kitamura C, Zurawel-Balaura L, Wong RK. How effective is video consultation in clinical oncology? A systematic review. Curr Oncol 2010;17:17-27.

14. Menachemi N, Burke DE, Ayers DJ. Factors affecting the adoption of

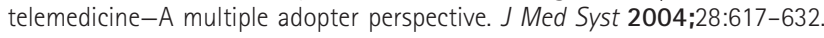

Address correspondence to:

Reena J. Salgia, MD

Henry Ford Hospital

2799 West Grand Boulevard, K7

Detroit, MI 48202

E-mail: rsalgia1@hfhs.org

Received: September 18, 2013

Revised: January 31, 2014

Accepted: February 5, 2014 


\section{This article has been cited by:}

1. Lisa M. Glass, Akbar K. Waljee, Heather McCurdy, Grace L. Su, Anne Sales. 2017. Specialty Care Access Network-Extension of Community Healthcare Outcomes Model Program for Liver Disease Improves Specialty Care Access. Digestive Diseases and Sciences 62:12, 3344-3349. [Crossref]

2. Elaine Scallan, Sarah Davis, Fred Thomas, Christine Cook, Kory Thomas, Patricia Valverde, Michael Kazanjian, Tim Byers. 2017. Supporting Peer Learning Networks for Case-Based Learning in Public Health: Experience of the Rocky Mountain Public Health Training Center With the ECHO Training Model. Pedagogy in Health Promotion 3:1_suppl, 52S-58S. [Crossref]

3. Sherry Ball, Brigid Wilson, Scott Ober, Ali Mchaourab. 2017. SCAN-ECHO for Pain Management: Implementing a Regional Telementoring Training for Primary Care Providers. Pain Medicine . [Crossref]

4. Carrol Zhou, Allison Crawford, Eva Serhal, Paul Kurdyak, Sanjeev Sockalingam. 2016. The Impact of Project ECHO on Participant and Patient Outcomes. Academic Medicine 91:10, 1439-1461. [Crossref]

5. Karla Thornton, Paulina Deming, Bruce Struminger, Miranda Sedillo, Eric Castillo, Miriam Komaromy, Sarah Zalud-Cerrato, Sanjeev Arora. 2016. Project ECHO: a Revolutionary Approach to Expanding Access to Modern Treatments for Hepatitis C. Current Hepatology Reports 15:3, 178-186. [Crossref]

6. Beste Lauren A., Mattox Elizabeth A., Pichler Raimund, Young Bessie A., Au David H., Kirsh Susan F., Germani Maureen W., Hedeen Ashley, Harp Bonnie K., Chang Michael F.. 2016. Primary Care Team Members Report Greater Individual Benefits from Long- Versus Short-Term Specialty Telemedicine Mentorship. Telemedicine and e-Health 22:8, 699-706. [Abstract] [Full Text HTML] [Full Text PDF] [Full Text PDF with Links]

7. Brian R. Wood, Kenton T. Unruh, Natalia Martinez-Paz, Mary Annese, Christian B. Ramers, Robert D. Harrington, Shireesha Dhanireddy, Lisa Kimmerly, John D. Scott, David H. Spach. 2016. Impact of a Telehealth Program That Delivers Remote Consultation and Longitudinal Mentorship to Community HIV Providers. Open Forum Infectious Diseases 3:3, ofw123. [Crossref]

8. Evan P. Carey, Joseph W. Frank, Robert D. Kerns, P. Michael Ho, Susan R. Kirsh. 2016. Implementation of telementoring for pain management in Veterans Health Administration: Spatial analysis. Journal of Rebabilitation Research and Development 53:1, 147-156. [Crossref] 